\title{
Descripción de la distribución espacial de la clorofila $a$, temperatura y salinidad en la plataforma y el talud continentales del Caribe centroamericano
}

\section{Description of chlorophyll $a$ spatial distribution, temperature and salinity on the continental shelf and slope of the Central American Caribbean}

\author{
Carlos Luis Brenes Rodríguez ${ }^{1}$, Rosario Benavides Morera ${ }^{1}$ y Sandra Loza Álvarez ${ }^{2}$
}

\begin{abstract}
RESUMEN
Con la coordinación regional de la Organización del Sector Pesquero y Acuícola del Istmo Centroamericano (OSPESCA), se llevó a cabo del 3 de enero al 3 de febrero del año 2011 una campaña de prospección pesquera sobre la plataforma y el talud continentales del Caribe centroamericano, a bordo del B/I Miguel Oliver. En 76 estaciones hidrográficas se determinaron la temperatura, salinidad y se estimó la concentración de clorofila $a$ través de un fluorómetro incorporado al CTD. La temperatura superficial varió entre 25 y $27^{\circ} \mathrm{C}$ y la salinidad en ese mismo nivel presentó valores entre 32.5 (Panamá) y 35.5 (Belice). La profundidad de la termoclina mostró una tendencia descendente de sur a norte localizándose entre los 140 y $155 \mathrm{~m}$. Los valores para la concentración de clorofila $a$ a se encuentran en el rango 0.01-1.01 $\mathrm{mg} \mathrm{m}^{-3}$. La distribución de la clorofila $a$ integrada en la capa de 0 a $150 \mathrm{~m}$ registró el valor más alto en las aguas panameñas $\left(25.25 \mathrm{mg} \mathrm{m}^{-2}\right)$, mientras la zona de Belice presentó el valor más bajo ( $\left.6.05 \mathrm{mg} \mathrm{m}^{-2}\right)$. Dos máximos de clorofila $a$ (zona de mezcla y estrato termoclino) caracterizan la zona sur del Caribe centroamericano (Costa Rica y Panamá) y un único máximo ubicado cerca de los $90 \mathrm{~m}$ sobresale en la distribución vertical de dicha variable entre Nicaragua y Belice. La concentración de clorofila $a$ promedio para todas las estaciones fue de $0.3 \mathrm{mg} \mathrm{m}^{-3}$, de modo que en la plataforma y el talud continentales predominan en general condiciones oligotróficas.
\end{abstract}

Palabras claves: Caribe, clorofila $a$, hidrografía, Centroamérica, plataforma continental.

\begin{abstract}
Under the regional coordination of OSPESCA (Central America Fisheries and Aquaculture Organization), an exploratory fishing survey campaign on the continental shelf and slope of the Central American Caribbean was conducted from January $3^{\text {rd }}$ to February $3^{\text {rd }}, 2011$ on board the Miguel Oliver R/V. Temperature and salinity were determined in 76 hydrographic stations, and chlorophyll $a$ concentration was estimated using a CTD fluorometer. Surface temperature ranged between $25^{\circ} \mathrm{C}$ and $27^{\circ} \mathrm{C}$ and salinity at that same level was between 32.5 (Panama) and 35.5 (Belize). The thermocline depth showed a descending tendency from south to north between 140 and $155 \mathrm{~m}$. Chlorophyll $a$ concentration ranged between 0.01 and $1.01 \mathrm{mg} \mathrm{m}^{-3}$. The distribution of chlorophyll $a$ in the 0 to $150 \mathrm{~m}$ layer was the highest in Panama $\left(25.25 \mathrm{mg} \mathrm{m}^{-2}\right)$ and the lowest in Belize $\left(6.05 \mathrm{mg} \mathrm{m}^{-2}\right)$. Two chlorophyll $a$ maximums (mixing layer and thermocline layer) characterize the southern area of the Central American Caribbean (Costa Rica and Panama), while only one maximum close to $90 \mathrm{~m}$ stands out in the vertical distribution of that variable between Nicaragua and Belize. Average chlorophyll $a$ concentration for all stations was $0.3 \mathrm{mg} \mathrm{m}^{-3}$; therefore, in general oligotrophic conditions stand out in the continental shelf and slope.
\end{abstract}

Keywords: Caribbean, chlorophyll $a$, hydrography, Central America, continental shelf.

\footnotetext{
Laboratorio de Oceanografía y Manejo Costero, Universidad Nacional, Heredia, Costa Rica, Apdo. 86-3000.

Departamento de Microorganismos y Necton, Instituto de Oceanología, Cuba. cbrenes.una@gmail.com*, rbmorera@gmail.com, sandra@oceano.inf.cu
}

Recibido: 1 de marzo de 2016

Corregido: 30 de setiembre de 2016

Aceptado: 19 de octubre de 2016

DOI: http://dx.doi.org/10.15359/revmar.9-1.3 


\section{INTRODUCCIÓN}

El Mar Caribe se localiza entre un grupo de islas pertenecientes a las Grandes Antillas al norte, las costas de Venezuela y Colombia al sur, las costas orientales de América Central al oeste y el arco de las Pequeñas Antillas al este. Tiene un área de $2640000 \mathrm{~km}^{2}$ y una profundidad media de $4400 \mathrm{~m}$ $\left(8^{\circ} \mathrm{N}-25^{\circ} \mathrm{N}, 85^{\circ} \mathrm{W}-55^{\circ} \mathrm{W}\right)$ (Jury, 2011).

Las costas orientales de América Central son parte de la porción extrema del Mar Caribe noroccidental. La plataforma continental de estas costas es relativamente angosta, excepto en Nicaragua y parte de Honduras. La orientación y el ancho de esta plataforma hacen suponer que existe una fuerte influencia oceánica sobre el comportamiento de las aguas costeras, y que muchos procesos que se originan fuera de la plataforma continental tienen su impacto directo sobre algunas propiedades físicas del agua de mar en zonas netamente litorales, en particular aquellos que modulan la circulación y sus procesos productivos (Brenes \& Castillo, 2000).

Las cartas de corrientes superficiales del Océano Atlántico tropical indican que el flujo resultante de la confluencia de la Corriente de Guyana y la Corriente Norecuatorial se bifurca justo antes de llegar a las Antillas Menores (Gallegos \& Czintron, 1997). Un flujo se dirige hacia el norte por fuera del arco antillano y se le denomina Corriente de Antillas. El otro flujo penetra al Mar Caribe a través de los diversos pasos entre las Antillas Menores y se le conoce como Corriente del Caribe (Johns et al. 2002). Pratt y Maul (2000) señalan como la principal fuente de variabilidad en la mesoescala de la Corriente del Caribe a la propagación de meandros y remolinos anticiclónicos. El giro ciclónico entre Panamá y Colombia, muy persistente en la circulación media del Mar Caribe, interactúa con las aguas que fluyen sobre la plataforma continental de Panamá, Colombia, Costa Rica y Nicaragua (Andrade et al. 2003).

Los vientos que soplan sobre el Mar Caribe fuerzan una circulación superficial particular en los primeros $200 \mathrm{~m}$ de la columna de agua, de manera que los movimientos ageostróficos que se observan en la corriente son el resultado del esfuerzo del viento. Cerca de la costa se producen interacciones con la plataforma y con movimientos a escala regional (Molinari et al. 1981).

Gallegos (1996) describe a través de diagramas T-S las distintas masas de agua que componen la columna por debajo de los $50 \mathrm{~m}$ en el Mar Caribe: el Agua Subsuperficial Subtropical del Atlántico Norte (ASSAN), el Agua Central del Atlántico Noroccidental (ACANO), el Agua Intermedia Antártica (AIA) y el Agua Profunda del Atlántico Norte (APAN).

Son escasas las zonas en los océanos donde se da una inyección de aguas intermedias ricas en nutrientes en los estratos superiores de la columna de agua. Una de estas zonas se localiza en el Mar Caribe, en el área sobre la plataforma continental de Nicaragua y Honduras, lo que ha permitido que 
esta región costera se caracterice por su gran variedad de recursos marinos (Gable, 1993), sustentando una flota pesquera artesanal importante.

Los organismos autótrofos se encuentran en la base de la trama trófica y desempeñan un papel muy importante en la producción primaria de los ecosistemas, ya que son capaces de elaborar materia orgánica mediante el proceso de fotosíntesis, donde intervienen diferentes pigmentos fotorreceptores (Jeffrey et al. 1997). La clorofila $a$ se encuentra presente en todos los organismos fotosintetizadores oxigénicos, por lo que su concentración en el agua de mar permite estimar la biomasa del fitoplancton e indirectamente la actividad biológica, siendo considerada una de las variables más importantes para describir la condición biológica promedio en los ecosistemas marinos (Manzano-Sarabia \& Salinas-Zavala, 2008).

Las mediciones de la temperaturay clorofila superficial del mar, obtenidas a partir de imágenes satelitales, han permitido la determinación de la distribución espacial de clorofila $a$ en la macroescala y son útiles en la estimación de la productividad biológica del océano, en los estudios biológico-pesqueros relacionados con el pronóstico de reclutamiento, supervivencia larval, áreas de desove, capturas y en el estudio de los cambios espacio-temporales en la abundancia de especies de interés comercial (Manzano-Sarabia \& Salinas-Zavala, 2008). No obstante, existe consenso entre varios autores de que dicha tecnología solo permite captar información de cerca del $20 \%$ del nivel superior de la columna de agua (Sathyendranath \& Platt, 1989; Falkowski \& Raven, 1997). Recientemente, Condal et al. (2013) realizaron un análisis de la variabilidad estacional anual y climatológica de la concentración de clorofila en tres regiones definidas por los autores como el Golfo de México (GM), el Caribe Occidental (CO) y las Bahamas (B), utilizando mapas de color del mar derivados de diversas fuentes de datos. La región CO incluye el Golfo de Honduras, el cual abarca los litorales de Belice, Guatemala y Honduras.

Con los avances tecnológicos de las últimas décadas, es cada vez más frecuente la estimación de la distribución vertical de clorofila a partir de datos de fluorescencia (Prairie et al. 2011). El trabajo más reciente que utiliza esta técnica en el Mar Caribe es el de Aguirre y Salmerón (2015), quienes presentan las características de fluorescencia para el Caribe Occidental en toda la columna de agua desde la superficie hasta los 200 $\mathrm{m}$ de profundidad.

El objetivo del presente trabajo fue estudiar el comportamiento de los campos de termohalinos y de clorofila (estimada a partir de datos de fluorescencia) a escala regional en el área ocupada por la plataforma y el talud continentales del Caribe de América Central, permitiendo complementar trabajos anteriores realizados en otras regiones del Mar Caribe. 


\section{METODOLOGÍA}

La campaña de muestreo se llevó a cabo a bordo del B/I Miguel Oliver de la Secretaría del Mar de España y se extendió del 3 de enero al 3 de febrero del año 2011. Se realizaron 76 estaciones hidrográficas (Fig. 1) con un perfilador vertical de temperatura, conductividad y profundidad (CTD) modelo SBE 25 SEALOGGER. El equipo contaba, adicionalmente, con un sensor para determinar fluorescencia. Las concentraciones de clorofila se obtuvieron a partir del siguiente algoritmo del fabricante:

$$
\mathrm{Chl}=\left(\mathrm{V}_{\text {muestra }}-\mathrm{V}_{\text {oscuro }}\right) * \text { factor de escala, }
$$

donde:

Chl es la concentración de clorofila de la muestra.

$\mathrm{V}_{\text {oscuro }}=0.05 \mathrm{~V}$ corresponde al voltaje para una muestra de agua de mar pura desionizada con ausencia total de luz.

$\mathrm{V}_{\text {muestra }}$ es el voltaje de la muestra de agua "in situ".

factor de escala $=12.35 \mathrm{mg} \mathrm{m}^{-3} \mathrm{~V}$.

Las concentraciones de clorofila $a$ fueron analizadas desde la superficie hasta los $200 \mathrm{~m}$ de profundidad. Por debajo de ese nivel las concentraciones eran despreciables.

El trabajo abarcó la plataforma y el talud continentales, de modo que el lance menos profundo alcanzó los 73 m y el de mayor profundidad, los 1474 $\mathrm{m}$. Las mediciones de las diferentes variables obtenidas por el CTD fueron posteriormente estandarizadas a cada metro de profundidad.
La profundidad de la termoclina se determinó a partir de la profundidad de la isoterma de $20^{\circ} \mathrm{C}$.

La presentación y posterior análisis de la información recolectada durante el crucero se hizo utilizando criterios de división política (por países), con el propósito de que las Oficinas Nacionales de Pesca de cada país integrantes de la OSPESCA pudieran tener una imagen lo más completa posible de sus recursos marinos (los cuales fueron desagregados en el Informe de Campaña por país) y de las características físicas del entorno.

Se utilizaron imágenes de satélite (con una resolución espacial de $1^{\circ} \mathrm{x} 1^{\circ}$ ) para obtener los campos superficiales promedios (enero 2011) de temperatura y clorofila $a$ en la zona de estudio y en las áreas adyacentes, a través del GES-DISC Interactive Online Visualization and Analysis Infrastructure, el Goddard Earth Sciences (GES) Data and Information Services Center (DISC) de la NASA (NOAA-NASA, 2016).

Los valores integrados de clorofila en la columna de agua están expresados como $\mathrm{mg} \mathrm{m}^{-2} \mathrm{y}$ se estimaron según la ecuación propuesta por Ramírez y Pizarro (2005): mg"clor $a$ " $\mathrm{m}^{-2}=\left(\left(\mathrm{d}_{\mathrm{n}+1}\right.\right.$ $\left.\left.-\mathrm{d}_{\mathrm{n}}\right)\left(\mathrm{C}_{\mathrm{n}+1}+\mathrm{C}_{\mathrm{n}}\right)\right) / 2+\left(\left(\mathrm{d}_{\mathrm{n}+2}-\mathrm{d}_{\mathrm{n}+1}\right)\left(\mathrm{C}_{\mathrm{n}+1}+\right.\right.$ $\left.\left.\mathrm{C}_{\mathrm{n}+2}\right)\right) / 2+\ldots \ldots$,

donde:

$\mathrm{C}=$ concentración de clorofila $a$ en cada profundidad

$\mathrm{d}=$ profundidad de muestreo $(\mathrm{m})$. 


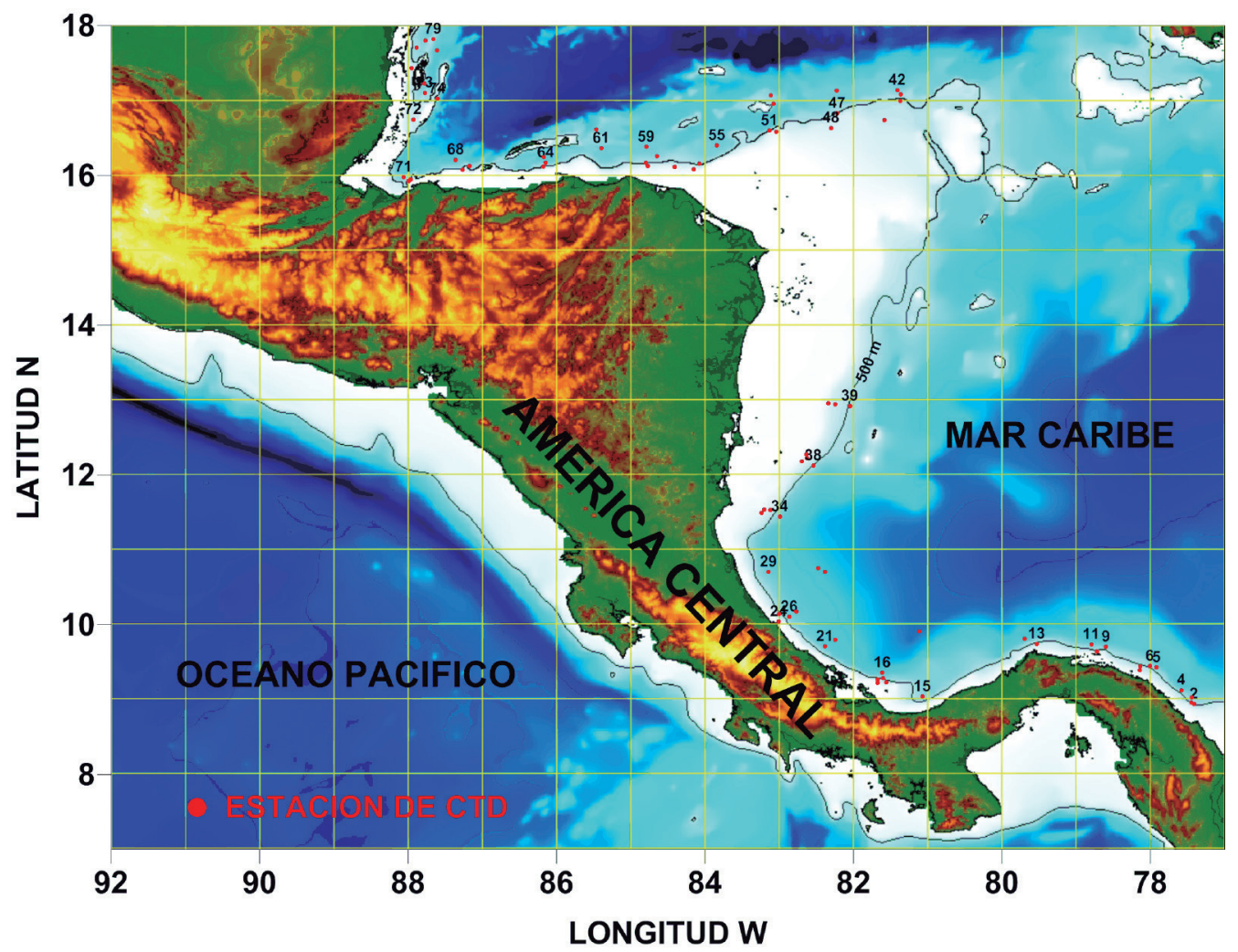

Fig. 1. Posición de las estaciones hidrográficas. Están enumeradas únicamente las que se mencionan en el texto

Fig. 1. Location of hydrographic stations. Only those mentioned in the text

\section{RESULTADOS}

Los campos superficiales de temperatura y concentración de clorofila $a$ para el período de muestreo se pueden observar en la Figura 2.

La región mostró valores de temperatura superficial entre 25 y $27^{\circ} \mathrm{C}$ (Fig. 2A). En el Mar Caribe, el mínimo estacional de esta variable ocurre entre diciembre y marzo. Durante este período los alisios del noreste soplan con mayor intensidad, y los fenómenos de afloramiento que ocurren sobre la plataforma continental de Nicaragua y Honduras suelen contribuir al enfriamiento del estrato superficial (Brenes et al. 1998).
Con respecto a la distribución superficial de clorofila $a$ (Fig. 2B), las zonas con mayores concentraciones se ubicaron en el área más cercana a la costa, con valores entre 2.5 y $10 \mathrm{mg} \mathrm{m}^{-3}$. En el borde de la plataforma y el talud continentales esas concentraciones disminuyeron drásticamente a valores del orden de $0.1 \mathrm{mg} \mathrm{m}^{-3}$.

El diagrama T-S para todos los lances de CTD se muestra en la Figura 3 y permitió identificar las diferentes masas de agua que ocupan la columna hasta el máximo nivel de muestreo.

Las figuras 4 y 5 presentan las distribuciones verticales de temperatura y 

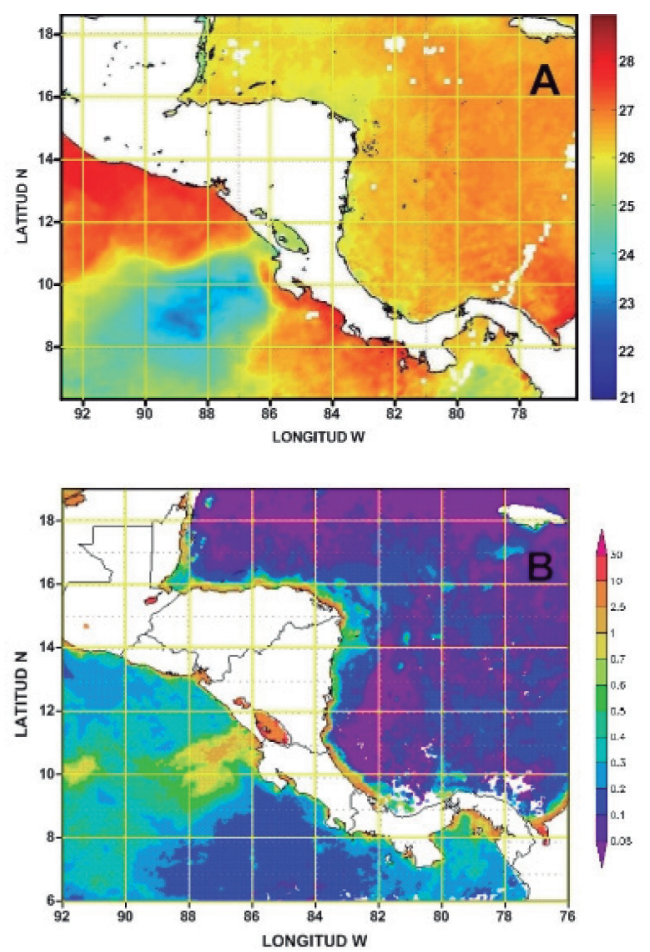

Fig. 2. Temperatura superficial promedio $\left({ }^{\circ} \mathrm{C}\right)(\mathrm{A})$ y clorofila $a\left(\mathrm{mg} \mathrm{m}^{-3}\right)(\mathrm{B})$ para enero del 2011. (Imágenes producidas utilizando el GES-DISC Interactive Online Visualization and Analysis Infrastructure, Goddard Earth Sciences (GES) Data and Information Services Center (DISC), NASA)

Fig. 2. Average surface temperature $\left({ }^{\circ} \mathrm{C}\right)$ (A) and chlorophyll $a\left(\mathrm{mg} \mathrm{m}^{-3}\right)$ (B) for January 2011. (Images produced using the GES-DISC Interactive Online Visualization and Analysis Infrastructure, Goddard Earth Sciences (GES) Data and Information Services Center (DISC), NASA)

salinidad para transectos paralelos a las costas de los diferentes países. En relación con la temperatura, por debajo de los $100 \mathrm{~m}$ de profundidad las variaciones del campo térmico fueron muy similares a lo largo de toda la región estudiada (Fig. 4). Las diferencias más evidentes se observan a nivel superficial, donde las aguas más cálidas se localizan en la parte sur de la plataforma continental caribeña en Panamá $\left(\mathrm{T}>27.5^{\circ} \mathrm{C}\right)$, mientras que en la región norte en Belice rondan $\operatorname{los} 26^{\circ} \mathrm{C}$ (Fig. 4A y E).

Las variaciones verticales en la profundidad promedio de la termoclina alcanzaron los 15 m (entre 140 y 155 m) y se observó una profundización de esta en la región norte del área (Honduras-Belice) (Cuadro 1, Fig. 4).

En el caso de la distribución vertical de la salinidad, las mayores variaciones se observaron en los niveles superiores de la columna de agua, donde las aguas menos salinas se localizaron en Panamá ( 30) correspondientes a los pares T-S menos salinos y más cálidos del diagrama T-S dispersivo (Fig. 3), y las de mayor concentración de sal se encontraron en Belice (35.5) (Figs. 3 y 5E).

Al analizar el comportamiento de la concentración de clorofila $a$ en los primeros $200 \mathrm{~m}$ de la columna de agua, se observó una disminución drástica de esta variable por debajo de los $100 \mathrm{~m}$, con valores entre 0.02 y $0.04 \mathrm{mg} \mathrm{m}^{-3}$ cerca de los $150 \mathrm{~m}$. A partir de esta profundidad comenzó a ser no detectable, razón por la cual su caracterización se realizó desde la superficie hasta los $150 \mathrm{~m}$.

En el caso de Panamá, la concentración de clorofila $a$ presentó valores entre $0.06 \mathrm{y} 1.01 \mathrm{mg} \mathrm{m}^{-3} \mathrm{y}$ un promedio de $0.24 \pm 0.04 \mathrm{mg} \mathrm{m}^{-3}$. Las mayores concentraciones se localizaron, en general, entre los 30 y $80 \mathrm{~m}$ de la columna de agua con valores de $0.27 \mathrm{y}$ $0.63 \mathrm{mg} \mathrm{m}^{-3}$, a excepción de la estación 4 


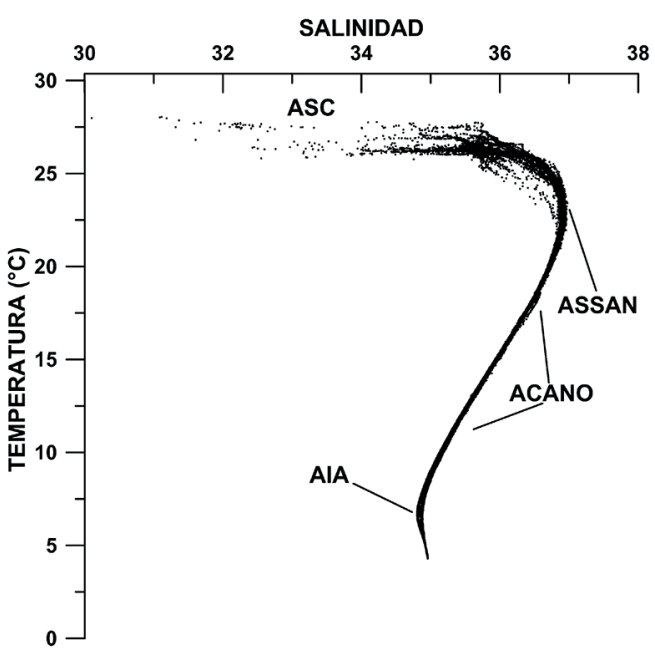

Fig. 3. Diagrama T-S dispersivo para todas las estaciones de CTD

Fig. 3. T-S Dispersion diagram for all CTD stations

Cuadro 1. Profundidad promedio de la termoclina referida como la profundidad a la cual se encuentra la isoterma de $20^{\circ} \mathrm{C}$

Table 1. Average thermocline depth, referred to as the depth of the isotherm at $20^{\circ} \mathrm{C}$

\begin{tabular}{cc}
\hline País & $\begin{array}{c}\text { Profundidad promedio de } \\
\text { la termoclina }(\mathbf{m})\end{array}$ \\
\hline Panamá & 140 \\
Costa Rica & 146 \\
Nicaragua & 142 \\
Honduras & 155 \\
Belice & 150 \\
\hline
\end{tabular}

donde se registró un valor máximo a los $15 \mathrm{~m}$ de $1.01 \mathrm{mg} \mathrm{m}^{-3}$ (Fig. 6A).

En la zona frente a Costa Rica, la concentración de clorofila $a$ promedio fue de $0.14 \pm 0.04 \mathrm{mg} \mathrm{m}^{-3}$, con un valor máximo de $0.71 \mathrm{mg} \mathrm{m}^{-3} \mathrm{y}$ un mínimo de $0.01 \mathrm{mg} \mathrm{m}^{-3}$. Entre los 30 y $60 \mathrm{~m}$, se registraron las mayores concentraciones $\left(0.21-0.71 \mathrm{mg} \mathrm{m}^{-3}\right)$, no obstante, esta variable presentó un comportamiento heterogéneo, ya que en algunas estaciones los máximos se localizaron entre los 8 y $17 \mathrm{~m}(0.22$ a $0.47 \mathrm{mg} \mathrm{m}^{-3}$ ) y en otras, entre los $80 \mathrm{y}$ $90 \mathrm{~m}\left(0.24\right.$ a $\left.0.44 \mathrm{mg} \mathrm{m}^{-3}\right)$ (Fig. 6B).

Para Nicaragua, el valor promedio de la concentración de clorofila $a$ fue de $0.20 \pm 0.06 \mathrm{mg} \mathrm{m}^{-3} \mathrm{y}$ los valores fluctuaron entre los 0.02 y $0.66 \mathrm{mg}$ $\mathrm{m}^{-3}$. Las mayores concentraciones (0.20-0.66 $\left.\mathrm{mg} \mathrm{m}^{-3}\right)$ se localizaron, en general, entre los 67 y 92 m (Fig. 6C).

La concentración de clorofila $a$ en la zona frente a Honduras estuvo entre 0.01 y $0.80 \mathrm{mg} \mathrm{m}^{-3}$, con un valor promedio de $0.20 \pm 0.06 \mathrm{mg} \mathrm{m}^{-3}$. Las mayores concentraciones, en general, se localizaron entre los 45 y $90 \mathrm{~m}$ de la columna de agua, oscilando entre los 0.18 y $0.76 \mathrm{mg} \mathrm{m}^{-3}$ (Fig. 6D).

Finalmente, en Belice la concentración de clorofila $a$ varió entre 0.01 y $0.52 \mathrm{mg} \mathrm{m}^{-3}(0.20 \pm 0.02$ $\left.\mathrm{mg} \mathrm{m}^{-3}\right)$. En esta zona, las mayores concentraciones se localizaron en el extremo norte y en los estratos más profundos, entre los 80 y $100 \mathrm{~m}$ de profundidad, mientras que en la región sur de Belice ese máximo se localizó a los $50 \mathrm{~m}$ con valores cercanos a 0.30 $\mathrm{mg} \mathrm{m}^{-3}$ (Fig. 6E).

El Cuadro 2 presenta la concentración de clorofila $a$ promedio por estratos en la columna de agua. En las aguas panameñas se registraron, en general, los mayores valores $(0.29$ $\left.\mathrm{mg} \mathrm{m}^{-3}\right)$. Se observó un incremento paulatino desde la superficie hasta los $100 \mathrm{~m}$, con un máximo centrado en los $50 \mathrm{~m}$ de profundidad. Por su parte, 
Cuadro 2. Distribución de la concentración de clorofila $a$ promedio en varias profundidades sobre la plataforma y el talud continentales de los diferentes países centroamericanos, en enero del 2011. (S/O sin observación)

Table 2. Distribution of mean chlorophyll $a$ at several depths on the continental shelf and slope of the different Central American countries, in January 2011 (S/O not observed)

\begin{tabular}{cccccc}
\hline Profundidad $(\mathbf{m})$ & \multicolumn{5}{c}{ Clorofila $\boldsymbol{a}\left(\mathbf{m g ~ m}^{-3}\right)$} \\
\hline $\mathbf{0}$ & Panamá & Costa Rica & Nicaragua & Honduras & Belice \\
$\mathbf{5}$ & 0.08 & $\mathrm{~S} / \mathrm{O}$ & $\mathrm{S} / \mathrm{O}$ & $\mathrm{S} / \mathrm{O}$ & $\mathrm{S} / \mathrm{O}$ \\
$\mathbf{1 0}$ & 0.15 & 0.17 & 0.09 & 0.15 & $\mathrm{~S} / \mathrm{O}$ \\
$\mathbf{1 5}$ & 0.18 & 0.16 & 0.07 & 0.08 & $\mathrm{~S} / \mathrm{O}$ \\
$\mathbf{2 0}$ & 0.21 & 0.12 & 0.10 & 0.08 & $\mathrm{~S} / \mathrm{O}$ \\
$\mathbf{2 5}$ & 0.21 & 0.12 & 0.10 & 0.08 & $\mathrm{~S} / \mathrm{O}$ \\
$\mathbf{5 0}$ & 0.22 & 0.13 & 0.11 & 0.07 & $\mathrm{~S} / \mathrm{O}$ \\
$\mathbf{7 5}$ & 0.29 & 0.14 & 0.08 & 0.13 & 0.02 \\
$\mathbf{1 0 0}$ & 0.27 & 0.14 & 0.16 & 0.23 & 0.07 \\
$\mathbf{1 2 5}$ & 0.23 & 0.12 & 0.23 & 0.23 & 0.25 \\
$\mathbf{1 5 0}$ & 0.11 & 0.08 & 0.08 & 0.08 & 0.09 \\
& 0.04 & 0.02 & 0.02 & 0.03 & 0.03 \\
\hline
\end{tabular}

en las aguas frente a Costa Rica los mayores valores se encontraron en los estratos subsuperficiales, mientras en el resto de los países de Centroamérica se presentaron en los estratos profundos.

En la Figura 7 se muestran los perfiles verticales de clorofila $a$, temperatura y densidad en estaciones ubicadas frente a los diferentes países. En la estación 5 frente a Panamá (Fig. 7A), el perfil vertical muestra la existencia de dos máximos de clorofila $a$, el primer máximo se localiza en la zona de mezcla con un valor de 0.46 $\mathrm{mg} \mathrm{m}^{-3}$ a los $33 \mathrm{~m}$ de profundidad. El segundo máximo se localiza en el nivel termoclino y alcanza un valor de 0.31 $\mathrm{mg} \mathrm{m} \mathrm{m}^{-3}$ a los $93 \mathrm{~m}$ de profundidad. En la estación 24 frente a Costa Rica (Fig. 7B), también se detectaron dos máximos de clorofila $a$ por encima de los $50 \mathrm{~m}$, con valores de $0.42 \mathrm{mg} \mathrm{m}^{-3}$

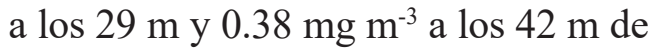
profundidad, mientras en las estaciones seleccionadas en las aguas de Nicaragua (34), Honduras (48) y Belice (74) solo se detectó el máximo de clorofila $a$ en el nivel de la termoclina $\left(0.22 \mathrm{mg} \mathrm{m}^{-3} \mathrm{a}\right.$ $\operatorname{los} 83 \mathrm{~m} ; 0.63 \mathrm{mg} \mathrm{m}^{-3}$ a los $80 \mathrm{~m}$ y 0.39 $\mathrm{mg} \mathrm{m}^{-3}$ a los $90 \mathrm{~m}$, respectivamente) (Fig. 7C, D y E).

El Cuadro 3 permite comparar los resultados obtenidos en el presente trabajo con reportes previos de las concentraciones de clorofila $a$ registradas por diferentes autores en áreas oceánicas de la cuenca del Caribe.

En el Cuadro 4 se presentan las concentraciones de clorofila integrada entre la superficie y los $150 \mathrm{~m}$ y por estratos (0-50 m y 50-150 m). 

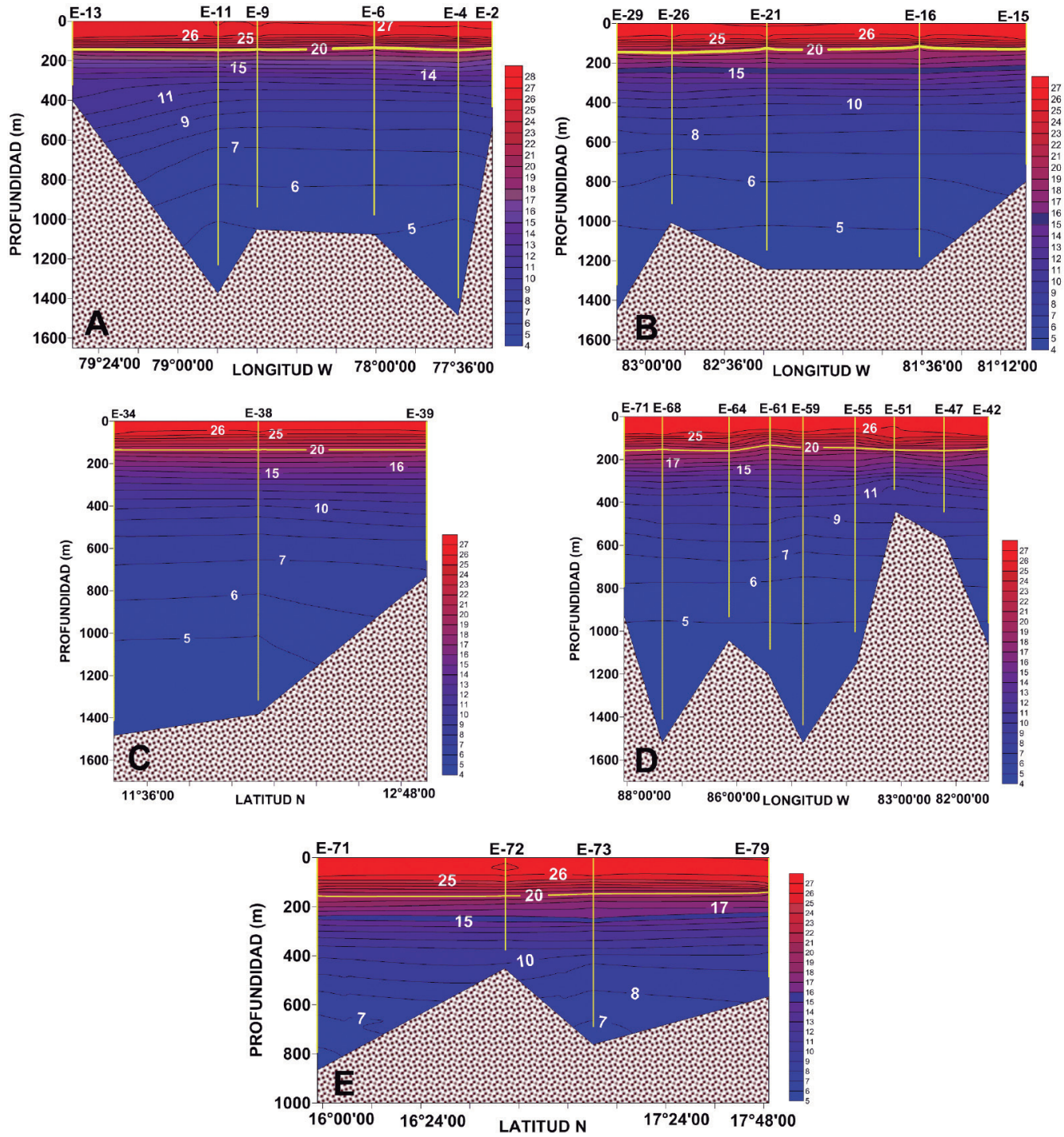

Fig. 4. Distribuciones verticales de temperatura $\left({ }^{\circ} \mathrm{C}\right)$ para transectos paralelos a la costa. Panamá (A), Costa Rica (B), Nicaragua (C), Honduras (D) y Belice (E). La isolínea de color amarillo representa la profundidad de la termoclina

Fig. 4. Vertical distribution of temperature $\left({ }^{\circ} \mathrm{C}\right)$ for transects along the coast. Panama (A), Costa Rica (B), Nicaragua (C), Honduras (D) and Belize (E). Yellow isoline represents thermocline depth

\section{DISCUSIÓN}

Las variaciones estacionales de los campos superficiales de temperatura en océanos y mares tropicales, como el Mar Caribe, son relativamente pequeñas, exceptuando aquellas áreas donde existen, por ejemplo, afloramientos costeros (Gallegos, 1996). Las temperaturas superiores a $26^{\circ} \mathrm{C}$ en los niveles menos profundos, obtenidas en el presente 
Cuadro 3. Concentraciones de clorofila $a$ en la columna de agua registradas por diferentes autores en áreas oceánicas de la cuenca del Caribe

Table 3. Chlorophyll $a$ concentrations recorded by different authors in oceanic areas of the Caribbean Basin

\begin{tabular}{llc}
\hline \multicolumn{1}{c}{ Autor } & \multicolumn{1}{c}{ Región } & Clorofila $\boldsymbol{a}\left(\mathbf{m g ~ m}^{-\mathbf{3}}\right)$ \\
\hline Harold et al. (1982) & Caribe en general & $0.053-0.154$ \\
Martinet \& Saint (1982) & Martinica & $0.020-0.580$ \\
Bianchi et al. (1995) & Golfo de México & $0.010-2.850$ \\
Webber y Roff (1996) & SE de Jamaica & $0.700-2.640$ \\
Campos-González et al. (2011) & Caribe Colombiano & $0.075-3.537$ \\
Aguirre y Salmerón (2015) & Caribe Occidental & $0.27-0.80$ \\
& Panamá & $0.06-1.01$ \\
& Costa Rica & $0.01-0.71$ \\
Presente estudio & Nicaragua & $0.02-0.66$ \\
& Honduras & $0.01-0.80$ \\
& Belice & $0.01-0.52$ \\
\hline
\end{tabular}

Cuadro 4. Concentración de clorofila $a$ integrada para varios estratos en los diferentes países del Caribe centroamericano

Table 4. Concentration of integrated chlorophyll $a$ for several strata in the different countries of the Central American Caribbean

\begin{tabular}{cccc}
\hline País & \multicolumn{3}{c}{ Concentración de clorofila $\boldsymbol{a}$ integrada $\left(\mathbf{m g ~ m}^{-2}\right)$} \\
\hline Panamá & $\mathbf{0 - 1 5 0 ~} \mathbf{~}$ & $\mathbf{0 - 5 0 ~} \mathbf{~ m}$ & $\mathbf{5 0 - 1 5 0 ~} \mathbf{~ m}$ \\
Costa Rica & 25.25 & 9.63 & 15.62 \\
Nicaragua & 20.38 & 7.34 & 13.04 \\
Honduras & 14.67 & 4.15 & 10.52 \\
Belice & 20.97 & 6.25 & 14.72 \\
\hline
\end{tabular}

trabajo, coinciden con las reportadas por otros autores y son representativas de la temporada en la cual los fuertes vientos del noreste inducen procesos de mezcla en las capas más someras y zonas de surgencias costeras (Gallegos, 1996; Brenes et al. 1998; Mooers \& Maul, 1998).
La capa de mezcla ya había sido descrita por Gallegos (1996) y Mooers \& Maul (1998) cuando propusieron visualizar la columna de agua en el Mar Caribe como un sistema de cuatro capas, y en este estudio ocupa un estrato térmicamente 

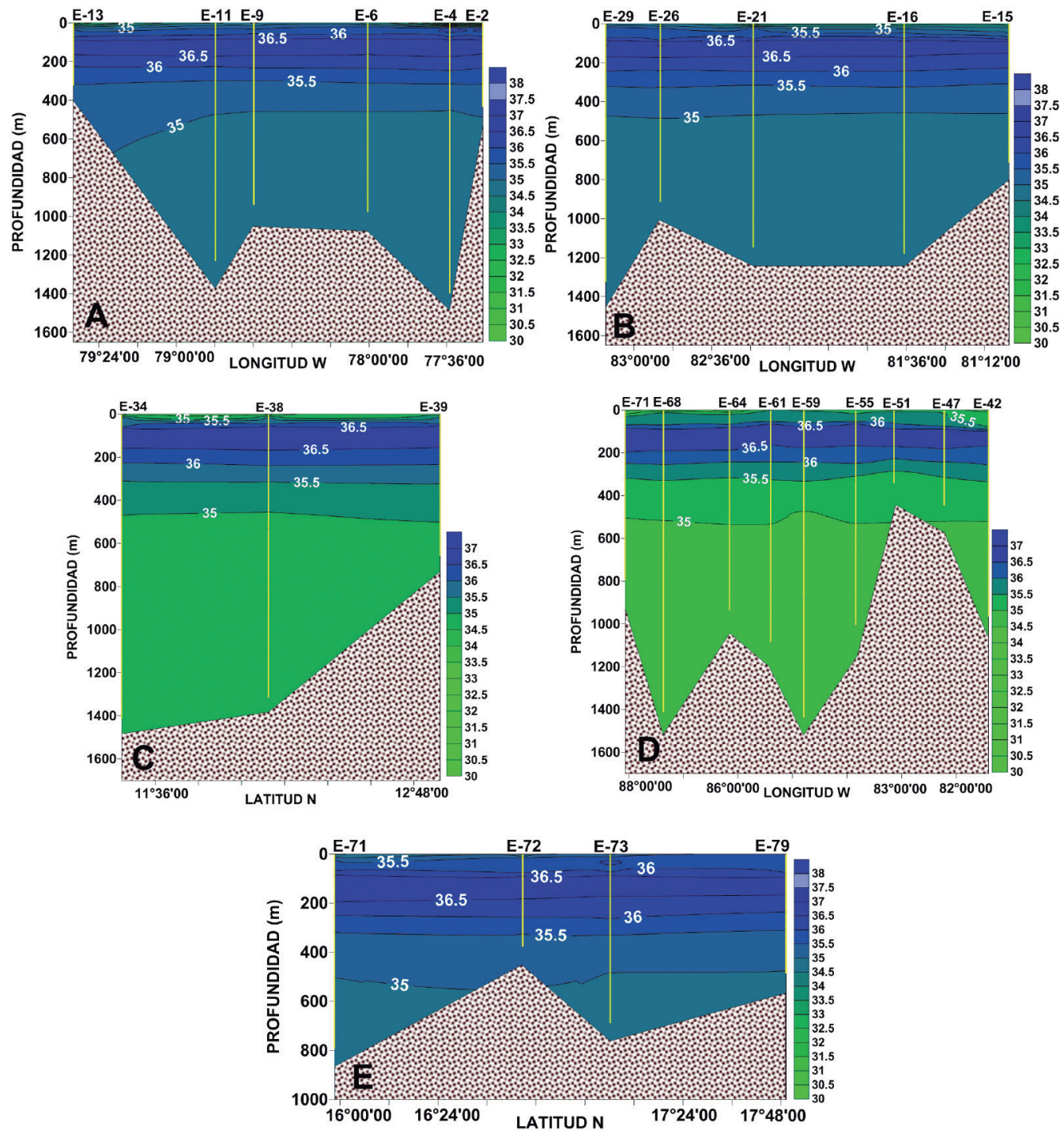

Fig. 5. Distribuciones verticales de salinidad (UPS) para transectos paralelos a la costa. Panamá (A), Costa Rica (B), Nicaragua (C), Honduras (D) y Belice (E)

Fig. 5. Vertical distribution of salinity (PSU) for transects along the coast. Panama (A), Costa Rica (B), Nicaragua (C), Honduras (D) and Belize (E)

homogéneo entre la superficie y los $100 \mathrm{~m}$ de profundidad.

Las aguas en estos niveles superiores son relativamente poco salinas $(\mathrm{S}<36)($ Fig. 5) y presentan al mismo tiempo las mayores variaciones a lo largo del Caribe centroamericano.
La profundización de la termoclina en el norte de la región estudiada (Cuadro 1) hace suponer que el afloramiento que caracteriza la región costera de Honduras, y que puede observarse en el extremo norte de la Figura 1, estaría restringido a 

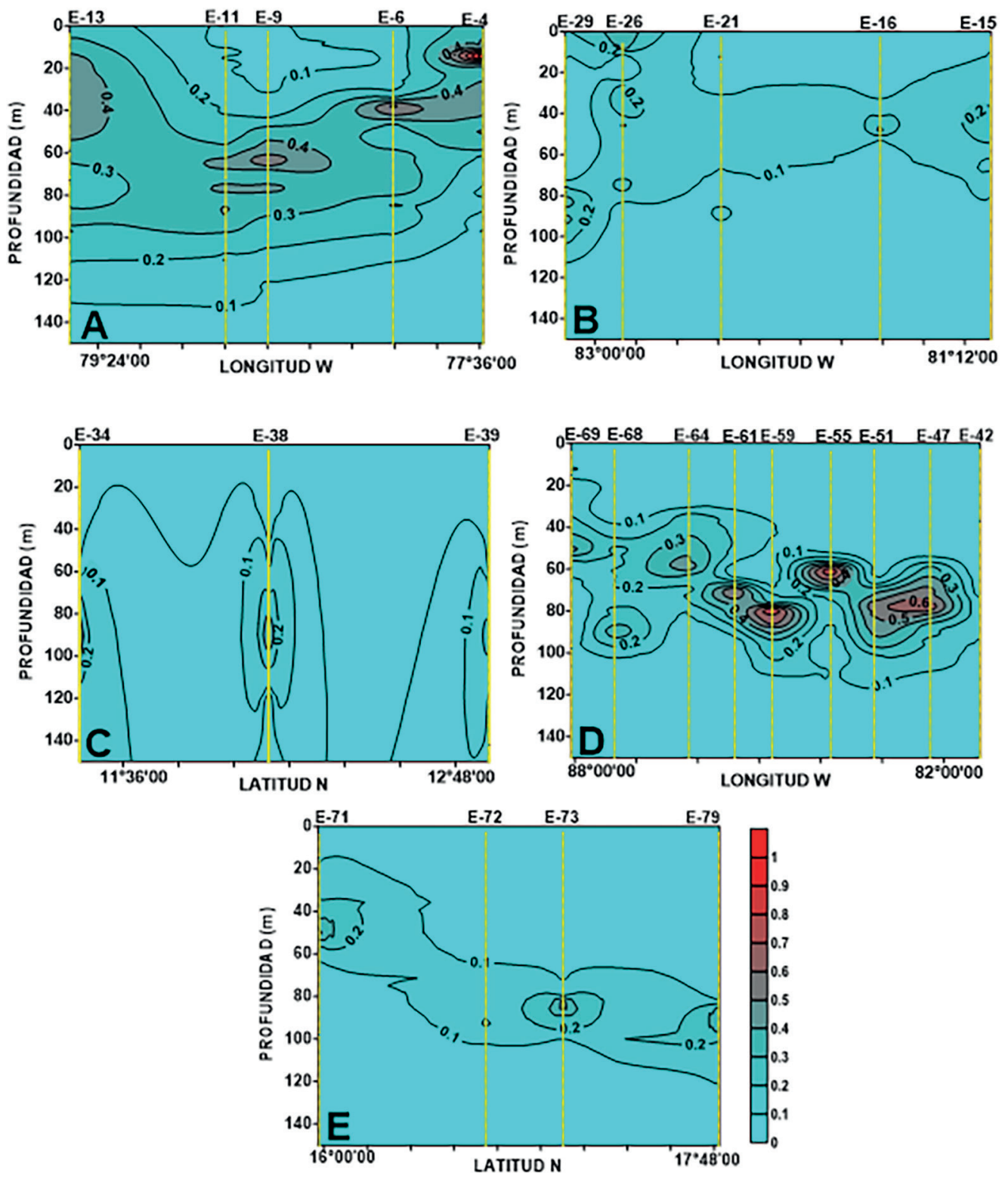

Fig. 6. Distribución vertical de la concentración de clorofila $a\left(\mathrm{mg} \mathrm{m}^{3}\right)$ en varios transectos ubicados frente a Panamá (A), Costa Rica (B), Nicaragua (C), Honduras (D) y Belice (E)

Fig. 6. Vertical distribution of chlorophyll $a$ concentration $\left(\mathrm{mg} \mathrm{m}^{3}\right)$ in several transects located off Panama (A), Costa Rica (B), Nicaragua (C), Honduras (D) and Belize (E)

las capas superficiales. No se observó ningún aumento significativo en las distribuciones de clorofila $a$ que

pudieran asociarse a un afloramiento fuerte en esta región.

El análisis de las propiedades 

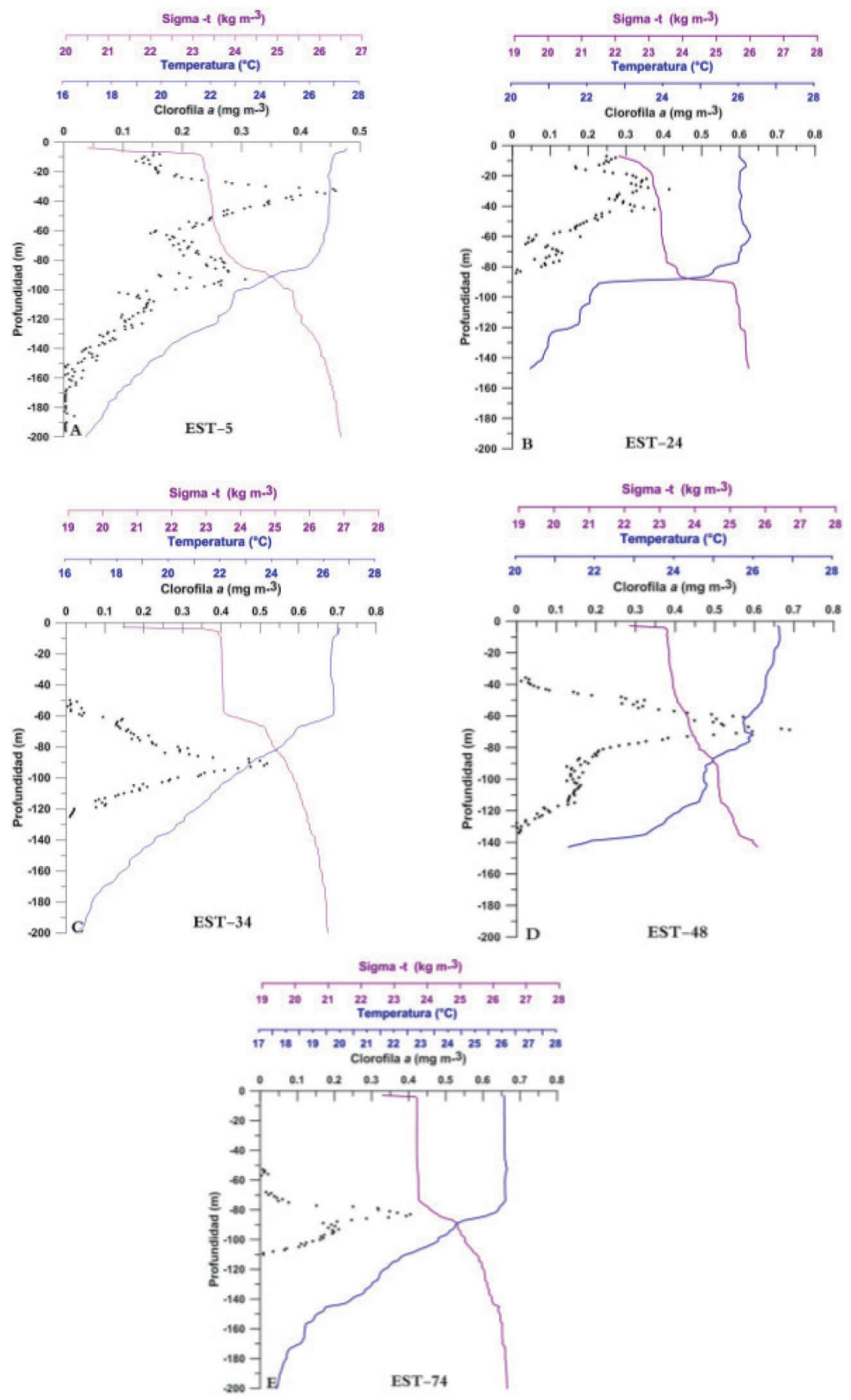

Fig. 7. Perfiles verticales de clorofila $a\left(\mathrm{mg} \mathrm{m}^{-3}\right)$ en estaciones ubicadas en el Caribe centroamericano. (A) Panamá, (B) Costa Rica, (C) Nicaragua, (D) Honduras y (E) Belice Fig. 7. Vertical profiles of chlorophyll $a\left(\mathrm{mg} \mathrm{m}^{3}\right)$ at stations located in the Central American Caribbean. (A) Panama, (B) Costa Rica, (C) Nicaragua, (D) Honduras and (E) Belize 
conservativas (temperatura y salinidad) a través del diagrama T-S dispersivo (Fig. 3) permitió identificar al Agua Superficial del Caribe (ASC) $\left(28^{\circ} \mathrm{C}<\right.$ $\mathrm{T}>30^{\circ} \mathrm{C}, 34.5<\mathrm{S}<36$ ) (Mooers \& Maul, 1998; Andrade \& Barton, 2000; Hernández-Guerra \& Joyce, 2000). Las bajas salinidades $(30<\mathrm{S}<34)$ fuera del rango del ASC provienen de las estaciones más costeras ubicadas en el extremo sur de la región estudiada, específicamente en aguas panameñas, y están asociadas a la presencia de una gran cantidad de ríos que desembocan en esa área (Sixaola, Changuinola, Teribe, Cricamola, Indio, Chagres y Gatún). Adicionalmente, la provincia de Bocas del Toro es la región que tiene los recursos hídricos más abundantes del Caribe de Panamá (Trejos, 2011). En enero del año 2011, según reportes presentados por la ACP (2013), todos estos ríos presentaron un aumento en sus caudales. Algunos ejemplos son: el río Chagres 161\%, Boquerón 115\%, Pequení 132\% y Gatún $153 \%$. Estos aumentos en los flujos estuvieron probablemente asociados a la intensificación de los empujes fríos entre diciembre y enero del 2011, relacionados con la presencia del fenómeno de La Niña en esa época. Hacia el norte, desde Honduras hasta Belice las salinidades superficiales muestran un aumento con valores superiores a 35.5 , debido a los afloramientos que ocurren en la plataforma continental de Honduras. La orientación este-oeste de la costa Caribe de Honduras y la componente del viento en esta misma dirección inducen un transporte de Ekman normal a la costa, responsable de los afloramientos costeros en esa zona (mayores salinidades y menores temperaturas superficiales) (Brenes et al. 1998).

En la misma Figura 3 y a partir de la clasificación propuesta por Gallegos (1996), se identificó adicionalmente el Agua Subsuperficial Subtropical del Atlántico Norte (ASSAN) $\left(21^{\circ} \mathrm{C}<\mathrm{T}<\right.$ $23^{\circ} \mathrm{C}, 36.6<\mathrm{S}<37$ ), su núcleo de alta salinidad se observa cerca de los $200 \mathrm{~m}$ de profundidad (Figs. 4 y 5). Por debajo de esta agua se encuentra el Agua Central del Atlántico Noroccidental (ACANO) hasta los $750 \mathrm{~m}\left(7^{\circ} \mathrm{C}<\right.$ $\mathrm{T}<20^{\circ} \mathrm{C}, 35<\mathrm{S}<36.7$ ) (Figs. 4 y 5). Finalmente, el Agua Intermedia Antártica (AIA) se extiende hasta los $950 \mathrm{~m}\left(2^{\circ} \mathrm{C}<\mathrm{T}<6^{\circ} \mathrm{C}\right)$ caracterizada por un mínimo salino cercano a 34.8. Los niveles de la columna de agua por debajo de los $1000 \mathrm{~m}$ de profundidad estarían ocupados por el Agua Profunda del Atlántico Norte (APAN), con temperaturas inferiores a $5^{\circ} \mathrm{C}$ y salinidades cercanas a 35 (Figs. 4 y 5).

Los valores para la concentración de clorofila $a$ se encuentran dentro del rango (0.01-3.537 mg $\mathrm{m}^{-3}$ ) reportado para la cuenca del Caribe por otros autores (Harold et al. 1982; Martinet \& Saint, 1982; Bianchi et al. 1995; Webber \& Roff, 1996; Campos-González et al. 2011; Aguirre \& Salmerón, 2015) (Cuadro 3). El incremento considerable observado en aguas panameñas podría estar relacionado con la influencia del giro ciclónico entre Panamá y Colombia, muy persistente en la circulación media del Mar Caribe (Andrade, 2001). 
La distribución vertical de la concentración de clorofila $a$ presentó dos patrones bien definidos (Fig. 7):

1. Una distribución bimodal en el extremo sur de la región (Costa Rica y Panamá), con un máximo en la capa de mezcla y otro al inicio del estrato termoclino. La existencia de los máximos de clorofila $a$ subsuperficial en aguas panameñas y de Costa Rica estarían asociadas a los aportes de aguas menos saladas y enriquecidas en nutrientes provenientes de la plataforma continental, que favorecen la actividad fotosintética y el incremento de la biomasa fitoplanctónica. Lo anterior se sustenta en los menores valores de salinidad registrados en el estrato superficial tanto en las aguas de Panamá como en las de Costa Rica con $\mathrm{S}<33$, las que podrían estar recibiendo la influencia de las descargas fluviales de la zona costera.

2. Una estructura con un único máximo profundo en el estrato termoclino (picnoclina) desde Nicaragua hasta Belice. Estos patrones de distribución vertical de la biomasa fitoplanctónica tienen implicaciones en las redes tróficas asociadas, al determinar la disponibilidad de alimento para el zooplancton herbívoro y filtrador (Signoret et al. 2006). La presencia de este máximo profundo de clorofila en el nivel termoclino (Fig. 7C, D y E) es una característica permanente que se localiza generalmente alrededor de la base de la zona eufótica y se distingue por una máxima actividad fotosintética (Signoret et al. 2006). En las aguas oceánicas adyacentes al área de estudio, Aguirre $\mathrm{y}$ Salmerón (2015) ubicaron ese máximo a una profundidad de 107 $\mathrm{m}$, mientras que en nuestro caso se localizó entre los 80 y $90 \mathrm{~m}$. Este rango coincide con los resultados reportados por Shcherbina et al. (2008), quienes lo ubicaron a 80 $\mathrm{m}$ cerca de las costas de Belice, permitiendo concluir al igual que Aguirre y Salmerón (2015) que la mayor parte del Caribe muestra una caracterización óptica similar a regiones de "aguas azules" o "mar abierto".

Este máximo profundo es un rasgo destacado de los perfiles verticales de clorofila $a$ en aguas oceánicas tropicales (Cullen, 1982), el cual supera entre cinco y ocho veces la concentración de clorofila superficial (Signoret et al. 1998), tal y como se encontró en el presente estudio. Estos perfiles se acoplan muy bien a la llamada "estructura tropical típica" descrita por Herbland y Voituriez (1979). Esta estructura es producto del acoplamiento entre los procesos físicos y biológicos en muchas regiones del Océano Atlántico Tropical, y se caracteriza en el perfil vertical por la asociación del máximo de clorofila, de la nutriclina y del máximo de producción primaria (Signoret et al. 1998). Takahashi y Hori (1984) encontraron que el picoplancton es una parte significativa de la biomasa 
fotosintética en este máximo profundo de clorofila en los trópicos.

McManus y Dawson (1994) al analizar los pigmentos de fitoplancton en el máximo de clorofila para el Mar Caribe y el Atlántico Occidental, encontraron que este máximo de forma aguda se localiza en la picnoclina o en la base de la capa de mezcla, coincidiendo con la parte superior de nutriclina. Nuestros resultados reportan un comportamiento similar (Fig. 7). Estos autores observaron también que el fitoplancton en este máximo está formado principalmente por proclorofitos picoplanctónicos. Resultados similares habían sido reportados para el Atlántico Norte (Olson et al. 1990; Veldhuis \& Kraay, 1990). Zubkov et al. (1998) encontraron que en aguas oligotróficas del Atlántico el género de cianobacterias fotosintéticas Prochlorococcus dominaba la biomasa del picofitoplancton, y que la profundidad ocupada por estas células aumentaba para aguas de esas características, ubicándose el máximo entre los 60 y 100 $\mathrm{m}$ de profundidad en la región tropical.

La influencia de varios factores físicos y biológicos y la magnitud del máximo profundo de la clorofila han sido analizadas mediante modelos físicobiologicos. Entre los factores biológicos se encuentran el zooplancton, que se alimenta de fitoplancton grande, y los heterótrofos, que ingieren fitoplancton pequeño $\mathrm{y}$ que afectan su magnitud, pero no su profundidad (Varela et al. 1992).

La concentración de clorofila $a$ promedio para todas las estaciones fue de $0.3 \mathrm{mg} \mathrm{m}^{-3}$, y según Tapia $\mathrm{y}$
Naranjo (2011) son aguas ligeramente productivas $\left(0.2<\mathrm{Chl}<0.5 \mathrm{mg} \mathrm{m}^{-3}\right)$. En la plataforma y el talud continentales predominan, en general, condiciones oligotróficas, resultado que coincide conlo reportado por Aguirre y Salmerón (2015), confirmando el hecho de que el Mar Caribe es considerado una región oligotrófica, donde el máximo profundo se observa en los estratos termoclinos (picnoclinos).

Al analizar la distribución de la clorofila $a$ integrada en la capa de 0 a $150 \mathrm{~m}$ (Cuadro 4), se evidencia que las aguas panameñas son las que registran el mayor valor de la producción de materia orgánica por unidad de superficie $\left(25.25 \mathrm{mg} \mathrm{m}^{-2}\right)$, mientras la zona de Belice fue la que presentó el valor más bajo $\left(6.05 \mathrm{mg} \mathrm{m}^{2}\right)$. Es importante señalar que de acuerdo con los valores de clorofila $a$ integrada para los estratos 0-50 m y 50-150 m, la mayor producción fitoplanctónica de estas aguas del Caribe centroamericano se concentra a partir de los $50 \mathrm{~m}$, con un importante incremento en Panamá (15.62 $\mathrm{mg} \mathrm{m}^{-2}$ ), seguido de Honduras, Costa Rica y Nicaragua $(14.72,13.04$ y $10.52 \mathrm{mg} \mathrm{m}^{-2}$, respectivamente), aunque se mantiene Belice con el valor más bajo $\left(5.78 \mathrm{mg} \mathrm{m}^{-2}\right)$ pero superior al del estrato 0-50 m (Cuadro 4).

\section{AGRADECIMIENTOS}

Este trabajo se llevó a cabo gracias al apoyo económico brindado por la Universidad Nacional a través del Proyecto Servicio Regional de Información Oceanográfica. Nuestro agradecimiento a Lic. Mario González 
Recinos, Director de OSPESCA, quien amablemente permitió la utilización de las bases de datos, y a todo el personal científico y la tripulación del B/I Miguel Oliver de la Secretaría General del Mar de España, que participaron en la Campaña de Investigación Pesquera Centroamérica-Caribe 2011.

\section{BIBLIOGRAFÍA}

ACP. Autoridad del Canal de Panamá. (2013). Anuario Hidrológico 2012. Ciudad de Panamá, Panamá: Departamento de ambiente, agua y energía. División de agua. Sección de recursos hídricos. Unidad de hidrología operativa.

Aguirre, R. \& Salmerón, O. (2015). Characterization of the western Caribbean Sea waters through in vivo chlorophyll fluorescence. Rev. Mar. Cost., 7, 9-26.

Andrade, C. (2001). Las corrientes superficiales en la cuenca de Colombia observadas con boyas de deriva. Rev. Acad. Colomb. Cienc., 25(96), 322-335.

Andrade, C. \& Barton, E. (2000). Eddy development and motion in the $\mathrm{Ca}$ ribbean Sea. J. Geophys. Res., 115(C11), 26191-26201. http://dx.doi. org/10.1029/2000JC000300

Andrade, C. A., Barton, E. D. \& Mooers, C. N. K. (2003). Evidence for an eastward flow along the Central and South American Caribbean Coast. J. Geophys. Res., 108(C6), 3185-3196. http:// dx.doi.org/10.1029/2002JC001549

Bianchi, T. S., Lambert, C. \& Biggs, D. C. (1995). Distribution of chlorophylla and phaeopigments in the northwestern Gulf of Mexico: A comparison between fluorimetric and high performance liquid chromatography measurements. Bull. Mar. Sci., 56, 25-32.
Brenes, C. L. \& Castillo, E. (2000). Caracterización hidrográfica de la laguna de Perlas, Nicaragua. Bluefields, Nicaragua: DIPAL II.

Brenes, C. L., Gallegos, A. \& Coen, E. (1998). Variación anual de la temperatura superficial en el golfo de Honduras. Rev. Biol. Trop., 46(4), 187-197.

Campos-González, M., Vargas-Castellanos, J. R., Franco-Herrera, A. \& Medina-Calderón, J. H. (2011). Distribución de la clorofila $a$ en las aguas adyacentes a las islas de Providencia y Santa Catalina, Caribe Colombiano. Bol. Invest. Mar. Cost., 40(2), 347-360. Condal, A., Vega-Moro, A. \& Ardisson, P. (2013). Climatological, annual, and seasonal variability in chlorophyll concentration in the Gulf of Mexico, western Caribbean, and Bahamas using NASA colour maps. Int. J. Rem. Sens., 34(5), 1591-1614. http://dx.doi.org/10. 1080/01431161.2012.723835

Cullen, J. J. (1982). The deep chlorophyll maximum: comparing vertical profiles of chlorophyll a. Can. J. Fish. Aquat. Sci., 39, 791-803. http://dx.doi. org $/ 10.1139 / \mathrm{f} 82-108$

Falkowski, P. G. \& Raven, J. A. (1997). Aquatic Photosynthesis. Oxford, United Kingdom: Blackwell Science.

Gable, F. (1993). Marine Habitats: Selected Environmental and Ecological Charts. In G. A. Maul (Ed.), Climatic Change in the Intra-Americas Sea (pp. 217-261). London, England: United Nations Environment Program.

Gallegos, A. (1996). Descriptive physical oceanography of the Caribbean Sea. In G. A. Maul (Ed.), Small Islands: Marine Science and Sustainable Development, Coast. Estuar. Stud (pp. 36-55). Washington D. C., EE. UU.: American Geophysical Union. 
Gallegos, A. \& Czintron, S. (1997). Aspectos de la oceanografía física regional del Mar Caribe. En M. F. Lavin (Ed.), Contribuciones a la Oceanografia Física en México, Monografía 3 (pp. 225-242). México, D. F.: Unión Geofísica Mexicana.

Harold, E., Marshall, H. \& Solder, J. (1982). Pelagic phytoplankton in the Caribbean Sea. Bull. Mar. Sci., 32(1), 354-365.

Herbland, A. \& Voituriez, B. (1979). Hydrological structure analysis for estimating the primary production in the tropical Atlantic Ocean. J. Mar. Res., 37, 87-101.

Hernández-Guerra, A. \& Joyce, T. M. (2000). Water masses and circulation in the surface layers of the Caribbean at $66^{\circ} \mathrm{W}$. Geophys. Res. Lett., 27(21), 3497-3500. http://dx.doi. org/10.1029/1999GL011230

Jeffrey, S. W., Mantoura, R. F. C. \& Wright, S. W. (1997). Phytoplankton pigments in oceanography: guidelines to modern methods. Paris, France: UNESCO Publishing.

Johns, W. E., Townsend, T. L., Fratantoni, D. M. \& Wilson, W. D. (2002). On the Atlantic inflow to the Caribbean Sea. Deep-Sea Res., I(49), 211-243. http://dx.doi.org/10.1016/ S0967-0637(01)00041-3

Jury, R. M. (2011). Environmental Influences on Caribbean Fish Catch. Int. Jour. Oceanogr., 2011, 1-11. http:// dx.doi.org/10.1155/2011/174729

Manzano-Sarabia, M. M. \& Salinas-Zavala, C. A. (2008). Variabilidad estacional e interanual de la concentración de clorofila $a$ y temperatura superficial del mar en la región occidental del Golfo de México: 1996-2007. Interciencia, 33(9), 628-634.

Martinet, J. \& Saint, C. (1982). Primary production of Atlantic waters of
Martinique coastal. Carib. J. Sci., 18, 103-105.

McManus, G. B. \& Dawson, R. (1994). Phytoplankton pigments in the deep chlorophyll maximum of the Caribbean Sea and the western tropical Atlantic Ocean. Mar. Ecol. Progr. Ser, 113, 199-206. http://dx.doi.org/10.3354/meps113199

Molinari, R. L., Spillane, M., Brooks, I., Atwood, D. \& Duckett, C. (1981). Surface currents in the Caribbean Sea as deduced from Lagrangian observation. J. Geophys. Res., 86(C7), 6537-6542. http://dx.doi.org/10.1029/ JC086iC07p06537

Mooers, C. N. K. \& Maul, G. A. (1998). Intra-Americas sea circulation, coastal segment (3, W). In A. Robinson \& K. H. Brink (Eds.), The Sea. Vol. 11 (pp. 183-208). New York, EE. UU.: John Wiley and Sons.

NOAA-NASA. (2016). GOES Project Science. Recuperado en septiembre 19, 2013 disponible en http://rsd.gsfc. nasa.gov/goes/

Olson, R. J., Chisholm, S. W., Zetrler, E. R., Altabet, M. \& Dusenberry, J. (1990). Spatial and temporal distributions of prochlorophyte picoplankton in the North Atlantic Ocean. Deep-Sea Res., 37(6), 1033-1051. http://dx.doi. org/10.1016/0198-0149(90)90109-9

Prairie, J. C., Franks, P. J., Jaffe, J. S., Doubell, M. J. \& Yamazaki, H. (2011). Physical and biological controls of vertical gradients in phytoplankton. Linmol. Oceanogr. Fluid \& Environ., 1(1), 75-90. doi:10.1215/215736981267403. http://dx.doi. org/10.1215/21573698-1267403

Pratt, R. W. \& Maul, G. A. (2000). Sea surface height variability of the IntraAmericas Sea from TOPEX/Poseidon 
satellite altimetry: 1992-1995. Bull. Mar. Sci., 67, 687-708.

Ramírez, B. \& Pizarro, E. (2005). Distribución de clorofila $a$ y feopigmentos en los canales australes chilenos comprendidos entre Puerto Montt y la Laguna de San Rafael, Chile. Cienc. Tecnol. Mar., 28(1), 45-62.

Sathyendranath, S. \& Platt, T. (1989). Remote sensing of ocean chlorophyll: Consequence of non-uniform pigment profile. Appl. Optics., 28(3), 490-495. http://dx.doi.org/10.1364/ AO.28.000490

Shcherbina, A. Y., Gawarkiewics, G. G., Linder, C. A. \& Thorrold, S. R. (2008). Mapping bathymetric and hydrographic features of Glover's Reef, Belize, with a REMUS autonomous underwater vehicle. Limnol. Oceanogr., 53(5, part 2), 2264-2272. http://dx.doi. org/10.4319/1o.2008.53.5_part_2.2264

Signoret, M., Bulit, C. \& Pérez, R. (1998). Patrones de distribución de clorofila $a$ y producción primaria en aguas del Golfo de México y del Mar Caribe. $\mathrm{Hi}$ drobiológica, 8(2), 81-88.

Signoret, M., Aldeco, J. \& Barreiro, M. T. (2006). Variabilidad de perfiles de clorofila $a$ obtenidos por fluorescencia natural en el oeste y centro del Golfo de México en junio de 1999. Hidrobiológica, 16(003), 221-232.

Takahashi, M. \& Hori, T. (1984). Abundance of picophytoplankton in the subsurface chlorophyll maximum layer in subtropical and tropical waters.
Mar. Biol., 79, 177-186. http://dx.doi. org/10.1007/BF00951826

Tapia, M. \& Naranjo, C. (2011). La clorofila $a$ como un indicador biológico en las estaciones fijas durante el evento La Niña 1999-2000. Acta Oceanográfica del Pacifico, 16(1), 25-31.

Trejos, N. (2011). Recursos hídricos Panamá 2011. Ciudad de Panamá: Centro del Agua.

Varela, R. A., Cruzado, A., Tintoré, J. \& García-Landona, E. (1992). Modeling the deep-chlorophyll maximum: A coupled physical-biological approach. J. Mar. Res., 50, 441-463. http://dx.doi. org/10.1357/002224092784797638

Veldhuis, M. \& Kraay, G. W. (1990). Vertical distribution and pigment composition of a picoplanktonic prochlorophyte in the subtropical North Atlantic: a combined study of HPLC analysis of pigments and flow cytometry. Mar. Ecol. Prog. Ser., 68, 121-127. http:// dx.doi.org/10.3354/meps068121

Webber, D. \& Roff, J. (1996). Influence of Kingston harbor on the phytoplankton community of the near shore Hellshire coast, southeast Jamaica. Bull. Mar. Sci., 59, 245-258.

Zubkov, M. A., Sleigh, M., Tarran, G. A., Burkill, P. H. \& Leakey, R. (1998). Picoplanktonic community structure on an Atlantic transect from 50 ${ }^{\circ} \mathrm{N}$ to $50^{\circ} \mathrm{S}$. Deep Sea Res., I(45), 1339-1355. http://dx.doi.org/10.1016/ S0967-0637(98)00015-6 